CRYSTALLOGRAPHIC COMMUNICATIONS

ISSN 2056-9890

Received 24 July 2019

Accepted 13 August 2019

Edited by J. Jasinski, Keene State College, USA

Keywords: crystal structure; chalcone; enone bridge; $\mathrm{O}-\mathrm{H}$... O interaction; data survey.

CCDC reference: 1946810

Supporting information: this article has supporting information at journals.iucr.org/e

\section{Crystal structure of $(E)$-3-(2-hydroxy-4-methyl- phenyl)-1-(2,4,6-trimethoxyphenyl)prop-2-en-1-one}

\author{
Maadh Jumaah, ${ }^{a}$ Huey Chong Kwong ${ }^{\mathrm{b}}$ and Melati Khairuddean ${ }^{\mathrm{a} *}$ \\ ${ }^{\mathbf{a}}$ School of Chemical Sciences, Universiti Sains Malaysia, Penang 11800 USM, Malaysia, and ${ }^{\mathbf{b}}$ Department of Chemistry, \\ Faculty of Science, Universiti Putra Malaysia, 43400 UPM Serdang, Selangor Darul Ehsan, Malaysia. *Correspondence \\ e-mail: melati@usm.my
}

The title chalcone derivative, $\mathrm{C}_{19} \mathrm{H}_{20} \mathrm{O}_{5}$, adopts a trans configuration with respect to the olefinic $\mathrm{C}=\mathrm{C}$ double bond. The 2-hydroxy-4-methylphenyl ring is coplanar with the attached enone bridge [torsion angle $=-179.96(14)^{\circ}$ ], where this plane is nearly perpendicular to the 2,4,6-trimethoxyphenyl ring [dihedral angle $\left.=75.81(8)^{\circ}\right]$. In the crystal, molecules are linked into chains propagating along [010] by an $\mathrm{O}-\mathrm{H} \cdots \mathrm{O}$ hydrogen bond. These chains are further connected into centrosymmetric dimer chains via weak $\mathrm{C}-\mathrm{H} \cdots \mathrm{O}$ interactions. The conformations of related chalcone derivatives are surveyed and all of these structures adopt a skeleton with two almost orthogonal aromatic rings.

\section{Chemical context}

Chalcones (1,3-diarylprop-2-en-1-ones) are precursors of flavonoids and isoflavonoids in the plant kingdom (Ni et al., 2004; Sahu et al., 2012). Structurally, they consist of two aryl groups linked by an $\alpha, \beta$-unsaturated ketone system (Ibrahim et al., 2012; Kumar et al., 2013), whereby the aryl groups can carry a variety of substituents such as hydroxyl, methoxy and alkenyl groups, which are by far the most commonly encountered ones in nature. With their structural simplicity and the associated ease of synthesis, chalcone compounds have attracted a considerable amount of attention because of their important pharmacological properties such as antioxidative (Aoki et al., 2008), anti-inflammatory (Israf et al., 2007), anti-gout (Jang et al., 2014), anti-histaminic (Yamamoto et al., 2004), anti-obesity (Birari et al., 2011), anti-protozoal (Chen et al., 1993), hypnotic (Cho et al., 2011) and anti-spasmodic (Sato et al., 2007) effects. In a continuation of our ongoing research on the properties of various chalcone derivatives (Sim et al., 2017, Kwong et al., 2018), we report herein the synthesis and crystal structure determination of the title compound, $\mathrm{C}_{19} \mathrm{H}_{20} \mathrm{O}_{5}$, (I).<smiles>COc1cc(OC)c(C(=O)/C=C/c2ccc(C)cc2O)c(OC)c1</smiles>

\section{Structural commentary}

The title chalcone derivative (I), crystallizes in the centrosymmetric triclinic space group $P \overline{1}$ and its asymmetric unit 


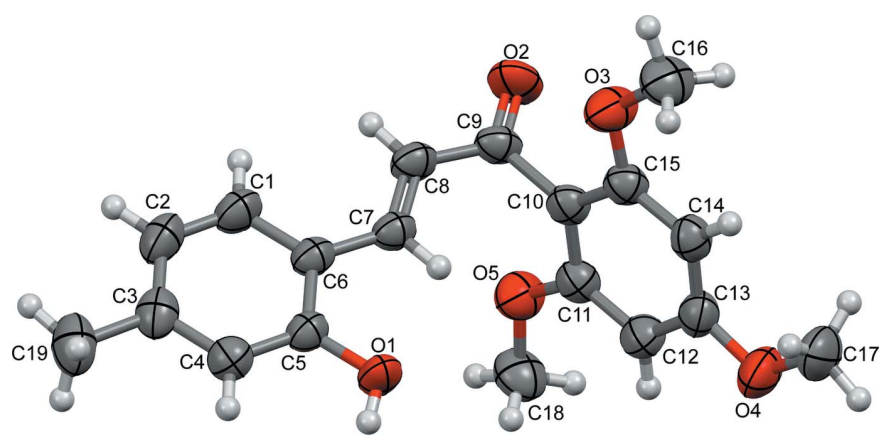

Figure 1

The title molecule with the atom-labelling scheme and displacement ellipsoids drawn at the $50 \%$ ellipsoid probability level.

consists of a single unique molecule (Fig. 1). This molecule is constructed of two substituted aromatic rings (2-hydroxy-4methylphenyl and 2,4,6-trimethoxyphenyl) and an enone $(-\mathrm{CH}=\mathrm{CH}-(\mathrm{C}=\mathrm{O})-$ ) connecting bridge. In the enone bridge, the $\mathrm{C} 6-\mathrm{C} 7, \mathrm{C} 8-\mathrm{C} 9$ and $\mathrm{C} 9-\mathrm{C} 10$ bond lengths of 1.446 (2), 1.441 (2) and 1.504 (2) A, respectively, confirm their single-bond character, whereas the $\mathrm{C} 7=\mathrm{C} 8$ and $\mathrm{C} 9=\mathrm{O} 2$ bond lengths of 1.340 (2) and 1.2255 (17) $\AA$, respectively, confirm the presence of a double bond. In addition, the $\mathrm{C} 6-\mathrm{C} 7-\mathrm{C} 8$ and $\mathrm{C} 8-\mathrm{C} 9-\mathrm{C} 10$ bond angles of $128.71(13)$ and $119.47(11)^{\circ}$, respectively, are consistent with the $s p^{2}$ hybridization of atoms C7, C8 and C9 (Kerr et al., 2001; LoghmaniKhouzani et al., 2009; Grealis et al., 2013). As the olefinic double bond $\mathrm{C} 7=\mathrm{C} 8$ adopts a trans configuration [C6-C7$\mathrm{C} 8-\mathrm{C} 9$ torsion angle $\left.=-179.96(14)^{\circ}\right]$, the structural conformation of (I) can be defined by three torsion angles. The torsion angles between the 2-hydroxy-4-methylphenyl ring and the olefinic double bond $\left(\mathrm{C} 5-\mathrm{C} 6-\mathrm{C} 7-\mathrm{C} 8, \tau_{1}\right)$, between the olefinic double bond and the carbonyl group $\left(\mathrm{C} 7-\mathrm{C} 8-\mathrm{C} 9-\mathrm{C} 10, \tau_{2}\right)$ and between the carbonyl group and the 2,4,6-trimethoxyphenyl ring $\left(\mathrm{C} 8-\mathrm{C} 9-\mathrm{C} 10-\mathrm{C} 11, \tau_{3}\right)$ are shown in Fig. 2. The torsion angles $\tau_{1}$ and $\tau_{2}$ are approximately $\pm 180^{\circ}$ or $0^{\circ}\left[\tau_{1}=-179.15(14)^{\circ}\right.$ and $\left.\tau_{2}=-0.8(2)^{\circ}\right]$, indicating that the 2-hydroxy-4-methylphenyl ring and the enone bridge are coplanar. In contrast, the carbonyl group is nearly perpendicular to the attached 2,4,6-trimethoxyphenyl ring, as $\tau_{3}$ is $76.87(19)^{\circ}$. In general, the molecule of (I) can be

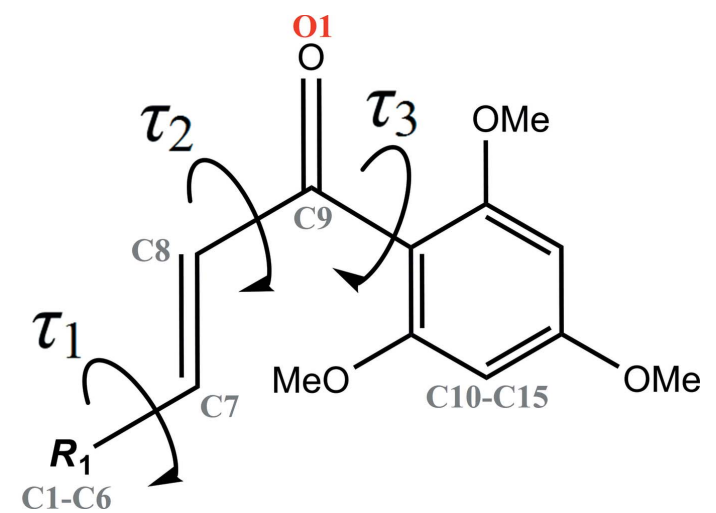

Figure 2

General chemical diagram showing torsion angles, $\tau_{1}, \tau_{2}$ and $\tau_{3}$.
Table 1

Hydrogen-bond geometry $\left(\AA,^{\circ}\right)$.

\begin{tabular}{lllll}
\hline$D-\mathrm{H} \cdots A$ & $D-\mathrm{H}$ & $\mathrm{H} \cdots A$ & $D \cdots A$ & $D-\mathrm{H} \cdots A$ \\
\hline $\mathrm{O} 1-\mathrm{H} 1 B \cdots \mathrm{O} 2^{\mathrm{i}}$ & 0.82 & 1.88 & $2.6653(15)$ & 161 \\
$\mathrm{C} 17-\mathrm{H} 17 A \cdots \mathrm{O} 1^{\mathrm{ii}}$ & 0.96 & 2.70 & $3.520(2)$ & 144 \\
\hline
\end{tabular}

Symmetry codes: (i) $x, y-1, z$; (ii) $-x+1,-y,-z$.

considered as two individual planes, the first comprising the 2-hydroxy-4-methylphenyl ring and the enone bridge [maximum deviation of 0.0021 (2) $\AA$ for atom C19], and the second the 2,4,6-trimethoxyphenyl ring [maximum deviation of 0.0059 (2) $\AA$ for atom C18]. These two mean planes form a dihedral angle of $75.84(4)^{\circ}$, hence the molecule of (I) possesses a skeleton with two almost orthogonal aromatic rings.

\section{Supramolecular features}

In the crystal, the molecules are linked into chains parallel to the $b$ axis via classical $\mathrm{O} 1-\mathrm{H} 1 B \cdots \mathrm{O} 2^{\mathrm{i}}$ hydrogen bonds (Fig. 3a). These chains are further connected into inversionrelated dimeric chains by weak $\mathrm{C} 17-\mathrm{H} 17 A \cdots \mathrm{O} 1^{\mathrm{ii}}$ intermolecular interactions (Fig. 3b, Table 1).

\section{Database survey}

A search of the Cambridge Structural Database (CSD version 5.40, last update May 2019; Groom et al., 2016) using (E)-3phenyl-1-(2,4,6-trimethoxyphenyl)prop-2-en-1-one as the reference moiety resulted in three chalcone structures containing 2,4,6-trimethoxyphenyl with different substituents.
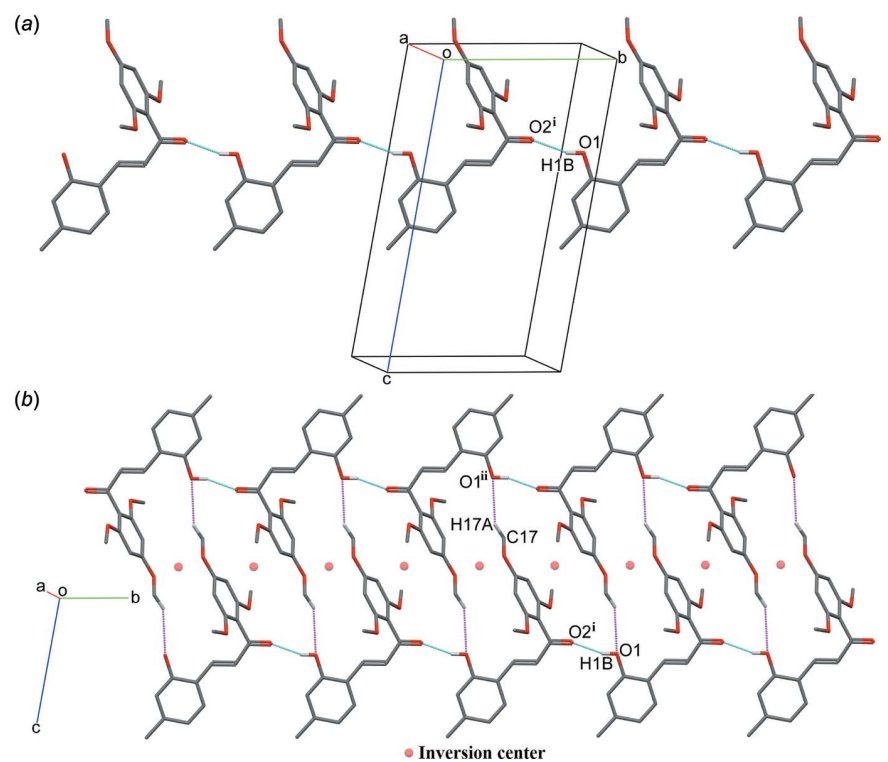

Figure 3

Views of $(a)$ a chain of molecules linked by $\mathrm{O}-\mathrm{H} \cdots \mathrm{O}$ hydrogen bonds (shown as cyan dotted lines) and $(b)$ a dimeric chain formed by weak $\mathrm{C}-$ $\mathrm{H} \cdot \mathrm{O}$ interactions (shown as magenta dotted lines). Symmetry code: (i) $x,-1+y, z$; (ii) $1-x,-y,-z$. Hydrogen atoms not involved in these interactions are omitted for clarity. 
Table 2

Selected torsion and dihedral angles $\left({ }^{\circ}\right)$.

The dihedral angle is that between the mean planes of the aromatic rings.

\begin{tabular}{|c|c|c|c|c|c|}
\hline Compound & $R_{1}$ & $\tau_{1}(\mathrm{C} 5-\mathrm{C} 6-\mathrm{C} 7-\mathrm{C} 8)$ & $\tau_{2}(\mathrm{C} 7-\mathrm{C} 8-\mathrm{C} 9-\mathrm{C} 10)$ & $\tau_{3}(\mathrm{C} 8-\mathrm{C} 9-\mathrm{C} 10-\mathrm{C} 11)$ & Dihedral angle \\
\hline (I) & 2-hydroxy-4-methylphenyl & $-179.2(1)$ & $-0.8(2)$ & $76.9(2)$ & $75.8(1)$ \\
\hline BAGXEN & 2,4,6-trimethoxyphenyl & 174.1 & -4.8 & 88.6 & 80.4 \\
\hline BUFMOF & 6-nitrobenzo $[d][1,3]$ dioxol-5-yl & 153.7 & 6.8 & 67.6 & 61.6 \\
\hline
\end{tabular}

Note: values for the minor occupancy component of GESRAZ are not given.

They include (E)-3-( $\left.\boldsymbol{R}_{\mathbf{1}}\right)$-1-(2,4,6-trimethoxyphenyl)prop-2en-1-one, where $\boldsymbol{R}_{\mathbf{1}}=2,4,6$-trimethoxyphenyl (BAGXEN; Kerr et al., 2001), 6-nitrobenzo[d][1,3]dioxol-5-yl (BUFMOF; Loghmani-Khouzani et al., 2009) and 4-methoxyphenyl (GESRAZ; Grealis et al., 2013). As in (I), the molecules of all these structures adopt a trans configuration with respect to $\mathrm{C}=\mathrm{C}$ double bond $(\mathrm{C} 6-\mathrm{C} 7-\mathrm{C} 8-\mathrm{C} 9$ torsion angles $=175.5-$ $179.1^{\circ}$ ). Although, $\tau_{1}$ for all of the structures indicates an antiperiplanar conformation (Table 2), in BUFMOF it deviates slightly from planarity $\left(\tau_{1}=152.7^{\circ}\right)$ whereas $\tau_{1}$ for the other molecules is approximately $180^{\circ}\left(\tau_{1}=174.1-176.0^{\circ}\right.$, Table 2$)$. Regarding the enone bridge, the torsion angle $\tau_{2}$ indicates that all of the structures are relatively planar $\left(\tau_{2}=-4.8-7.6^{\circ}\right)$. The torsion angles $\tau_{3}$ always almost indicate a perpendicular arrangement $\left(\tau_{3}=67.6-88.6^{\circ}\right)$. This might arise from the steric repulsion between the carbonyl group and the attached 2,4,6trimethoxyphenyl ring. This results in an overall L-shape for all of the structures, with the dihedral angle between the mean planes of the two aromatic rings being $61.6-80.4^{\circ}$.

\section{Synthesis and crystallization}

A reaction scheme for the synthesis of the title compound is given in Fig. 4. A solution of trimethoxyacetophenone ( $2 \mathrm{mmol})$ in $20 \mathrm{~mL} \mathrm{MeOH}$, LiOH (2.4 mmol) and 2-hydroxy4-methylbenzaldehyde $(1.6 \mathrm{mmol})$ was stirred at $368 \mathrm{~K}$ and the reaction progress was monitored by TLC. The reaction was quenched with diluted hydrochloric acid to $\mathrm{pH}=6$ and extracted with ethyl acetate. The organic layer was washed with aqueous $\mathrm{NaHCO}_{3}$, water, and brine, successively. The organic layer was dried over anhydrous $\mathrm{Na}_{2} \mathrm{SO}_{4}$ and then concentrated to provide the product as a brown powder. The residue was purified by column chromatography with petroleum ether/ethyl acetate (10:1) as an eluent to afford the target compound (Yan et al., 2016). Slow evaporation from an acetonitrile-water mixture provided X-ray quality crystals for the target chalcone compound.
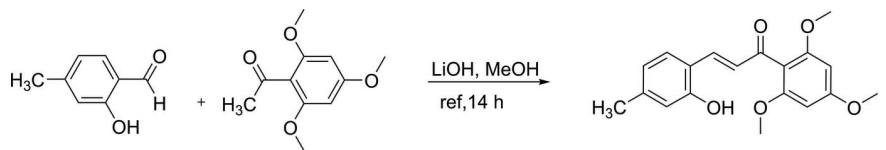

Figure 4

Reaction scheme for the synthesis of the title chalcone.
(E)-3-(2-hydroxy-4-methylphenyl)-1-(2,4,6-trimethoxyphenyl)prop-2-en-1-one (I)

Brown powder, yield 84.1\%. m.p. 503-506 K. IR $\left(\mathrm{cm}^{-1}\right)$ : $3283(\mathrm{O}-\mathrm{H}), 2929$ and $2842\left(\mathrm{Csp}^{3}-\mathrm{H}\right), 1603(\mathrm{C}=\mathrm{O}), 1586$ and $1457(\mathrm{C}=\mathrm{C}) .{ }^{1} \mathrm{H}$ NMR $\left(500 \mathrm{MHz}, \mathrm{DMSO}-d_{6}\right) \delta$, ppm: $10.02(s, 1 \mathrm{H}), 7.45(d, J=16.2 \mathrm{~Hz}, 1 \mathrm{H}), 7.44(d, J=7.0 \mathrm{~Hz}, 1 \mathrm{H})$, $6.86(d, J=16.2 \mathrm{~Hz}, 1 \mathrm{H}), 6.65(d, J=8.0 \mathrm{~Hz}, 2 \mathrm{H}), 6.30(s, 2 \mathrm{H})$, $3.83(s, 3 \mathrm{H}), 3.70(s, 6 \mathrm{H}), 2.23(s, 3 \mathrm{H}) .{ }^{13} \mathrm{C}-\mathrm{NMR}(125 \mathrm{MHz}$, DMSO- $\left.d_{6}\right) \delta$, ppm: $194.3,162.2,158.4,157.1,142.5,140.2$, 128.8, 127.9, 121.1, 118.9, 117.0, 111.9, 91.6, 56.2, 55.9, 21.6. CHN Elemental analysis: Calculated for $\mathrm{C}_{19} \mathrm{H}_{20} \mathrm{O}_{5}: \mathrm{C}, 69.50$; H, 6.14; N. Found: C, 67.81; H, 5.72; N, 0.00.

\section{Refinement}

Crystal data, data collection and structure refinement details are summarized in Table 3. C-bound $\mathrm{H}$ atoms were positioned

Table 3

Experimental details.

\begin{tabular}{|c|c|}
\hline Crystal data & \\
\hline Chemical formula & $\mathrm{C}_{19} \mathrm{H}_{20} \mathrm{O}_{5}$ \\
\hline$M_{\mathrm{r}}$ & 328.35 \\
\hline Crystal system, space group & Triclinic, $P \overline{1}$ \\
\hline Temperature (K) & 296 \\
\hline$a, b, c(\AA)$ & $6.8072(3), 8.5792(4), 15.8010(7)$ \\
\hline$\alpha, \beta, \gamma\left({ }^{\circ}\right)$ & $100.365(1), 99.433(1), 104.984(1)$ \\
\hline$V\left(\AA^{3}\right)$ & $855.09(7)$ \\
\hline$Z$ & 2 \\
\hline Radiation type & Mo $K \alpha$ \\
\hline$\mu\left(\mathrm{mm}^{-1}\right)$ & 0.09 \\
\hline Crystal size $(\mathrm{mm})$ & $0.57 \times 0.25 \times 0.21$ \\
\hline Data collection & \\
\hline Diffractometer & $\begin{array}{l}\text { Bruker APEXII DUO CCD area- } \\
\text { detector }\end{array}$ \\
\hline Absorption correction & $\begin{array}{l}\text { Multi-scan (SADABS; Bruker, } \\
\text { 2012) }\end{array}$ \\
\hline$T_{\min }, T_{\max }$ & $0.908,0.950$ \\
\hline $\begin{array}{l}\text { No. of measured, independent and } \\
\text { observed }[I>2 \sigma(I)] \text { reflections }\end{array}$ & $33448,5018,3199$ \\
\hline 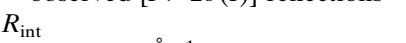 & 0.030 \\
\hline$(\sin \theta / \lambda)_{\max }\left(\AA^{-1}\right)$ & 0.706 \\
\hline Refinement & \\
\hline$R\left[F^{2}>2 \sigma\left(F^{2}\right)\right], w R\left(F^{2}\right), S$ & $0.052,0.171,1.05$ \\
\hline No. of reflections & 5018 \\
\hline No. of parameters & 217 \\
\hline $\mathrm{H}$-atom treatment & $\mathrm{H}$-atom parameters constrained \\
\hline$\Delta \rho_{\max }, \Delta \rho_{\min }\left(\mathrm{e} \AA^{-3}\right)$ & $0.27,-0.19$ \\
\hline
\end{tabular}

Computer programs: APEX2 and SAINT (Bruker, 2012), SHELXS97 (Sheldrick, 2008), SHELXL2013 (Sheldrick, 2015), Mercury (Macrae et al., 2006) and PLATON (Spek, 2009). 
geometrically $(\mathrm{C}-\mathrm{H}=0.93-0.96 \AA)$ and refined using a riding model with $U_{\text {iso }}(\mathrm{H})=1.2 U_{\text {eq }}(\mathrm{C})$ or $1.5 U_{\text {eq }}(\mathrm{C}-$ methyl $)$. The Obound hydrogen was located from difference-Fourier maps and refined freely with $\mathrm{O}-\mathrm{H}=0.82 \AA$.

\section{Funding information}

The authors would like to express their sincere gratitude to Universiti Sains Malaysia for the financial support from the Bridging Research Grant 2017 (304.PKIMIA.6316171).

\section{References}

Aoki, N., Muko, M., Ohta, E. \& Ohta, S. (2008). J. Nat. Prod. 71, 1308-1310.

Birari, R. B., Gupta, S., Mohan, C. G. \& Bhutani, K. K. (2011). Phytomedicine, 18, 795-801.

Bruker (2012). APEX2, SAINT and SADABS. Bruker AXS Inc., Madison, Wisconsin, USA.

Chen, M., Christensen, S. B., Blom, J., Lemmich, E., Nadelmann, L., Fich, K., Theander, T. G. \& Kharazmi, A. (1993). Antimicrob. Agents Chemother. 37, 2550-2556.

Cho, S., Kim, S., Jin, Z., Yang, H., Han, D., Baek, N. I., Jo, J., Cho, C. W., Park, J. H., Shimizu, M. \& Jin, Y. H. (2011). Biochem. Biophys. Res. Commun. 413, 637-642.

Grealis, J. P., Müller-Bunz, H., Ortin, Y., Casey, M. \& McGlinchey, M. J. (2013). Eur. J. Org. Chem. 2013, 332-347.

Groom, C. R., Bruno, I. J., Lightfoot, M. P. \& Ward, S. C. (2016). Acta Cryst. B72, 171-179.

Ibrahim, M., AlRefai, M., Abu-El-Halawa, R., Tashtoush, H., Alsohaili, S. \& Masad, M. (2012). Jordan. J. Chem. 7, 115-123.
Israf, D. A., Khaizurin, T. A., Syahida, A., Lajis, N. H. \& Khozirah, S. (2007). Mol. Immunol. 44, 673-679.

Jang, I. T., Hyun, S. H., Shin, J. W., Lee, Y. H., Ji, J. H. \& Lee, J. S. (2014). Mycobiology. 42, 296-300.

Kerr, P. J., Pyke, S. M., Ward, A. D. \& Tiekink, E. R. T. (2001). Z. Kristallogr. New Cryst. Struct. 216, 601.

Kumar, C. S., Loh, W. S., Ooi, C. W., Quah, C. K. \& Fun, H. K. (2013). Molecules, 18, 11996-12011.

Kwong, H. C., Rakesh, M. S., Chidan Kumar, C. S., Maidur Shivaraj, R., Patil Parutagouda, S., Quah Ching, K., Win, Y.-F., Parlak, C. \& Chandraju, S. (2018). Z. Kristallogr. Cryst. Mater. 233, 349-360.

Loghmani-Khouzani, H., Abdul Rahman, N., Robinson, W. T., Yaeghoobi, M. \& Kia, R. (2009). Acta Cryst. E65, o2545.

Macrae, C. F., Edgington, P. R., McCabe, P., Pidcock, E., Shields, G. P., Taylor, R., Towler, M. \& van de Streek, J. (2006). J. Appl. Cryst. 39, 453-457.

Ni, L., Meng, C. Q. \& Sikorski, J. A. (2004). Expert Opin. Ther. Pat. 14, 1669-1691.

Sahu, N. K., Balbhadra, S. S., Choudhary, J. \& Kohli, D. V. (2012). Curr. Med. Chem. 19, 209-225.

Sato, Y., He, J. X., Nagai, H., Tani, T. \& Akao, T. (2007). Biol. Pharm. Bull. 30, 145-149.

Sheldrick, G. M. (2008). Acta Cryst. A64, 112-122.

Sheldrick, G. M. (2015). Acta Cryst. C71, 3-8.

Sim, A., Chidan Kumar, C. S., Kwong, H. C., Then, L. Y., Win, Y.-F., Quah, C. K., Naveen, S., Chandraju, S., Lokanath, N. K. \& Warad, I. (2017). Acta Cryst. E73, 896-900.

Spek, A. L. (2009). Acta Cryst. D65, 148-155.

Yamamoto, T., Yoshimura, M., Yamaguchi, F., Kouchi, T., Tsuji, R., Saito, M., Obata, A. \& Kikuchi, M. (2004). Biosci. Biotechnol. Biochem. 68, 1706-1711.

Yan, J., Chen, J., Zhang, S., Hu, J., Huang, L. \& Li, X. (2016). J. Med. Chem. 59, 5264-5283. 


\section{supporting information}

Acta Cryst. (2019). E75, 1379-1382［https://doi.org/10.1107/S2056989019011289]

\section{Crystal structure of (E)-3-(2-hydroxy-4-methylphenyl)-1-(2,4,6-trimethoxy-} phenyl)prop-2-en-1-one

\section{Maadh Jumaah, Huey Chong Kwong and Melati Khairuddean}

Computing details

Data collection: APEX2 (Bruker, 2012); cell refinement: SAINT (Bruker, 2012); data reduction: SAINT (Bruker, 2012); program(s) used to solve structure: SHELXS97 (Sheldrick, 2008); program(s) used to refine structure: SHELXL2013 (Sheldrick, 2015); molecular graphics: SHELXL2013 (Sheldrick, 2015) and Mercury (Macrae et al., 2006); software used to prepare material for publication: SHELXL2013 (Sheldrick, 2015) and PLATON (Spek, 2009).

(E)-3-(2-Hydroxy-4-methylphenyl)-1-(2,4,6-trimethoxyphenyl)prop-2-en-1-one

\section{Crystal data}

$\mathrm{C}_{19} \mathrm{H}_{20} \mathrm{O}_{5}$

$M_{r}=328.35$

Triclinic, $P \overline{1}$

$a=6.8072(3) \AA$

$b=8.5792(4) \AA$

$c=15.8010(7) \AA$

$\alpha=100.365(1)^{\circ}$

$\beta=99.433(1)^{\circ}$

$\gamma=104.984(1)^{\circ}$

$V=855.09(7) \AA^{3}$

\section{Data collection}

Bruker APEXII DUO CCD area-detector diffractometer

Radiation source: fine-focus sealed tube Graphite monochromator $\varphi$ and $\omega$ scans

Absorption correction: multi-scan

(SADABS; Bruker, 2012)

$T_{\min }=0.908, T_{\max }=0.950$

Refinement

Refinement on $F^{2}$

Least-squares matrix: full

$R\left[F^{2}>2 \sigma\left(F^{2}\right)\right]=0.052$

$w R\left(F^{2}\right)=0.171$

$S=1.05$

5018 reflections

217 parameters

0 restraints
$Z=2$

$F(000)=348$

$D_{\mathrm{x}}=1.275 \mathrm{Mg} \mathrm{m}^{-3}$

Mo $K \alpha$ radiation, $\lambda=0.71073 \AA$

Cell parameters from 6606 reflections

$\theta=2.5-25.7^{\circ}$

$\mu=0.09 \mathrm{~mm}^{-1}$

$T=296 \mathrm{~K}$

Block, brown

$0.57 \times 0.25 \times 0.21 \mathrm{~mm}$

33448 measured reflections

5018 independent reflections

3199 reflections with $I>2 \sigma(I)$

$R_{\text {int }}=0.030$

$\theta_{\text {max }}=30.1^{\circ}, \theta_{\min }=1.3^{\circ}$

$h=-9 \rightarrow 9$

$k=-12 \rightarrow 12$

$l=-22 \rightarrow 22$

Hydrogen site location: inferred from neighbouring sites

$\mathrm{H}$-atom parameters constrained

$w=1 /\left[\sigma^{2}\left(F_{\mathrm{o}}^{2}\right)+(0.0776 P)^{2}+0.1331 P\right]$

where $P=\left(F_{\mathrm{o}}{ }^{2}+2 F_{\mathrm{c}}{ }^{2}\right) / 3$

$(\Delta / \sigma)_{\max }<0.001$

$\Delta \rho_{\max }=0.27 \mathrm{e} \AA^{-3}$

$\Delta \rho_{\min }=-0.19$ e $\AA^{-3}$ 


\section{Special details}

Geometry. All esds (except the esd in the dihedral angle between two 1.s. planes) are estimated using the full covariance matrix. The cell esds are taken into account individually in the estimation of esds in distances, angles and torsion angles; correlations between esds in cell parameters are only used when they are defined by crystal symmetry. An approximate (isotropic) treatment of cell esds is used for estimating esds involving l.s. planes.

Fractional atomic coordinates and isotropic or equivalent isotropic displacement parameters $\left(\AA^{2}\right)$

\begin{tabular}{|c|c|c|c|c|}
\hline & $x$ & $y$ & $z$ & $U_{\text {iso }} * / U_{\text {eq }}$ \\
\hline $\mathrm{O} 1$ & $0.64130(18)$ & $0.04792(12)$ & $0.33273(7)$ & $0.0582(3)$ \\
\hline H1B & 0.622234 & -0.051206 & 0.330607 & $0.087^{*}$ \\
\hline $\mathrm{O} 2$ & $0.5594(2)$ & $0.71937(13)$ & $0.28620(9)$ & $0.0750(4)$ \\
\hline $\mathrm{O} 3$ & $0.82818(19)$ & $0.58546(15)$ & $0.17162(8)$ & $0.0718(4)$ \\
\hline $\mathrm{O} 4$ & $0.3336(2)$ & $0.11733(16)$ & $-0.04471(7)$ & $0.0741(4)$ \\
\hline O5 & $0.18978(18)$ & $0.33623(15)$ & $0.22860(8)$ & $0.0653(3)$ \\
\hline $\mathrm{C} 1$ & $0.7621(3)$ & $0.3898(2)$ & $0.52362(11)$ & $0.0587(4)$ \\
\hline $\mathrm{H} 1 \mathrm{~A}$ & 0.765072 & 0.500561 & 0.536913 & $0.070^{*}$ \\
\hline $\mathrm{C} 2$ & $0.8168(3)$ & $0.3175(2)$ & $0.59089(11)$ & $0.0636(4)$ \\
\hline $\mathrm{H} 2 \mathrm{~A}$ & 0.856694 & 0.379893 & 0.648810 & $0.076^{*}$ \\
\hline $\mathrm{C} 3$ & $0.8134(2)$ & $0.1518(2)$ & $0.57349(10)$ & $0.0576(4)$ \\
\hline $\mathrm{C} 4$ & $0.7548(2)$ & $0.0621(2)$ & $0.48690(10)$ & $0.0536(4)$ \\
\hline $\mathrm{H} 4 \mathrm{~A}$ & 0.752792 & -0.048531 & 0.474238 & $0.064^{*}$ \\
\hline $\mathrm{C} 5$ & $0.6988(2)$ & $0.13422(17)$ & $0.41849(9)$ & $0.0462(3)$ \\
\hline C6 & $0.7017(2)$ & $0.30075(17)$ & $0.43524(9)$ & $0.0461(3)$ \\
\hline $\mathrm{C} 7$ & $0.6399(2)$ & $0.37021(17)$ & $0.36199(9)$ & $0.0462(3)$ \\
\hline $\mathrm{H} 7 \mathrm{~A}$ & 0.600214 & 0.297697 & 0.306638 & $0.055^{*}$ \\
\hline C8 & $0.6322(2)$ & $0.52507(17)$ & $0.36314(10)$ & $0.0530(4)$ \\
\hline H8A & 0.670606 & 0.601882 & 0.417082 & $0.064^{*}$ \\
\hline $\mathrm{C} 9$ & $0.5668(2)$ & $0.57758(17)$ & $0.28428(10)$ & $0.0506(3)$ \\
\hline $\mathrm{C} 10$ & $0.5066(2)$ & $0.45590(17)$ & $0.19626(10)$ & $0.0474(3)$ \\
\hline $\mathrm{C} 11$ & $0.3145(2)$ & $0.33383(18)$ & $0.16927(10)$ & $0.0496(3)$ \\
\hline $\mathrm{C} 12$ & $0.2590(2)$ & $0.2222(2)$ & $0.08827(10)$ & $0.0553(4)$ \\
\hline $\mathrm{H} 12 \mathrm{~A}$ & 0.130137 & 0.141133 & 0.070877 & $0.066^{*}$ \\
\hline $\mathrm{C} 13$ & $0.3996(3)$ & $0.23348(19)$ & $0.03331(10)$ & $0.0539(4)$ \\
\hline C14 & $0.5911(2)$ & $0.35440(19)$ & $0.05781(10)$ & $0.0528(4)$ \\
\hline H14A & 0.683280 & 0.361673 & 0.020255 & $0.063^{*}$ \\
\hline $\mathrm{C} 15$ & $0.6426(2)$ & $0.46422(18)$ & $0.13936(10)$ & $0.0502(3)$ \\
\hline $\mathrm{C} 16$ & $0.9764(3)$ & $0.6078(3)$ & $0.11824(14)$ & $0.0747(5)$ \\
\hline H16A & 1.098130 & 0.697163 & 0.149806 & $0.112^{*}$ \\
\hline H16B & 1.014327 & 0.507669 & 0.103655 & $0.112 *$ \\
\hline $\mathrm{H} 16 \mathrm{C}$ & 0.917128 & 0.633866 & 0.065061 & $0.112 *$ \\
\hline $\mathrm{C} 17$ & $0.4721(4)$ & $0.1217(3)$ & $-0.10396(12)$ & $0.0798(6)$ \\
\hline H17A & 0.408006 & 0.034279 & -0.156077 & $0.120^{*}$ \\
\hline H17B & 0.501338 & 0.226820 & -0.119882 & $0.120^{*}$ \\
\hline $\mathrm{H} 17 \mathrm{C}$ & 0.599756 & 0.106887 & -0.075655 & $0.120^{*}$ \\
\hline $\mathrm{C} 18$ & $-0.0021(3)$ & $0.2085(3)$ & $0.21029(14)$ & $0.0741(5)$ \\
\hline H18A & -0.072792 & 0.225952 & 0.257352 & $0.111 *$ \\
\hline H18B & -0.087708 & 0.210054 & 0.155938 & $0.111^{*}$ \\
\hline
\end{tabular}




$\begin{array}{lllll}\text { H18C } & 0.024368 & 0.102923 & 0.205183 & 0.111^{*} \\ \text { C19 } & 0.8715(3) & 0.0699(3) & 0.64692(12) & 0.0785(6) \\ \text { H19A } & 0.908022 & 0.148913 & 0.702583 & 0.118^{*} \\ \text { H19B } & 0.755129 & -0.021899 & 0.646508 & 0.118^{*} \\ \text { H19C } & 0.988302 & 0.030616 & 0.638320 & 0.118^{*}\end{array}$

Atomic displacement parameters $\left(\AA^{2}\right)$

\begin{tabular}{|c|c|c|c|c|c|c|}
\hline & $U^{11}$ & $U^{22}$ & $U^{33}$ & $U^{12}$ & $U^{13}$ & $U^{23}$ \\
\hline $\mathrm{O} 1$ & $0.0834(8)$ & $0.0397(5)$ & $0.0461(6)$ & $0.0191(5)$ & $0.0077(5)$ & $0.0007(4)$ \\
\hline $\mathrm{O} 2$ & $0.1156(11)$ & $0.0394(6)$ & $0.0798(8)$ & $0.0294(6)$ & $0.0388(8)$ & $0.0135(5)$ \\
\hline $\mathrm{O} 3$ & $0.0660(7)$ & $0.0665(7)$ & $0.0692(8)$ & $-0.0007(6)$ & $0.0305(6)$ & -0.0029 (6) \\
\hline O4 & $0.0803(9)$ & $0.0796(8)$ & $0.0461(6)$ & $0.0084(7)$ & $0.0123(6)$ & $-0.0022(6)$ \\
\hline O5 & $0.0577(7)$ & $0.0702(7)$ & $0.0659(7)$ & $0.0118(5)$ & $0.0271(6)$ & $0.0090(6)$ \\
\hline $\mathrm{C} 1$ & $0.0631(9)$ & $0.0534(8)$ & $0.0508(8)$ & $0.0140(7)$ & $0.0116(7)$ & $-0.0047(7)$ \\
\hline $\mathrm{C} 2$ & $0.0594(9)$ & $0.0766(11)$ & $0.0441(8)$ & $0.0141(8)$ & $0.0094(7)$ & $-0.0018(7)$ \\
\hline $\mathrm{C} 3$ & $0.0434(8)$ & $0.0790(11)$ & $0.0486(8)$ & $0.0153(7)$ & $0.0102(6)$ & $0.0146(7)$ \\
\hline $\mathrm{C} 4$ & $0.0527(8)$ & $0.0538(8)$ & $0.0543(8)$ & $0.0169(7)$ & $0.0119(7)$ & $0.0113(7)$ \\
\hline $\mathrm{C} 5$ & $0.0454(7)$ & $0.0463(7)$ & $0.0431(7)$ & $0.0118(6)$ & $0.0099(6)$ & $0.0030(6)$ \\
\hline C6 & $0.0432(7)$ & $0.0440(7)$ & $0.0464(7)$ & $0.0097(5)$ & $0.0117(6)$ & $0.0018(6)$ \\
\hline $\mathrm{C} 7$ & $0.0476(7)$ & $0.0406(7)$ & $0.0466(7)$ & $0.0112(5)$ & $0.0130(6)$ & 0.0009 \\
\hline $\mathrm{C} 8$ & $0.0607(9)$ & $0.0413(7)$ & $0.0523(8)$ & $0.0129(6)$ & $0.0163(7)$ & $-0.0009(6)$ \\
\hline C9 & $0.0586(8)$ & $0.0357(6)$ & $0.0600(9)$ & $0.0137(6)$ & $0.0246(7)$ & $0.0074(6)$ \\
\hline $\mathrm{C} 10$ & $0.0557(8)$ & $0.0403(7)$ & $0.0507(8)$ & $0.0185(6)$ & $0.0173(6)$ & $0.0108(6)$ \\
\hline $\mathrm{C} 11$ & $0.0525(8)$ & $0.0491(8)$ & $0.0530(8)$ & $0.0198(6)$ & $0.0169(6)$ & $0.0152(6)$ \\
\hline $\mathrm{C} 12$ & $0.0529(8)$ & $0.0563(9)$ & $0.0522(8)$ & $0.0117(7)$ & $0.0082(7)$ & $0.0113(7)$ \\
\hline $\mathrm{C} 13$ & $0.0638(9)$ & $0.0550(8)$ & $0.0426(7)$ & $0.0206(7)$ & $0.0073(7)$ & $0.0103(6)$ \\
\hline $\mathrm{C} 14$ & $0.0597(9)$ & $0.0568(8)$ & $0.0475(8)$ & $0.0214(7)$ & $0.0192(7)$ & $0.0136(7)$ \\
\hline C15 & $0.0539(8)$ & $0.0446(7)$ & $0.0543(8)$ & $0.0156(6)$ & $0.0169(7)$ & $0.0113(6)$ \\
\hline $\mathrm{C} 16$ & $0.0663(11)$ & $0.0762(12)$ & 0.0799 (13) & 0.0099 (9) & $0.0345(10)$ & $0.0137(10)$ \\
\hline $\mathrm{C} 17$ & $0.1025(15)$ & $0.0791(12)$ & $0.0497(10)$ & $0.0168(11)$ & $0.0256(10)$ & $0.0018(8)$ \\
\hline $\mathrm{C} 18$ & $0.0613(10)$ & $0.0826(13)$ & $0.0820(13)$ & $0.0132(9)$ & $0.0271(9)$ & $0.0297(10)$ \\
\hline C19 & $0.0684(11)$ & $0.1119(16)$ & $0.0570(11)$ & $0.0267(11)$ & $0.0087(9)$ & $0.0291(10)$ \\
\hline
\end{tabular}

Geometric parameters $\left(\AA,{ }^{o}\right)$

\begin{tabular}{llll}
\hline $\mathrm{O} 1-\mathrm{C} 5$ & $1.3613(16)$ & $\mathrm{C} 8-\mathrm{H} 8 \mathrm{~A}$ & 0.9300 \\
$\mathrm{O} 1-\mathrm{H} 1 \mathrm{~B}$ & 0.8200 & $\mathrm{C} 9-\mathrm{C} 10$ & $1.504(2)$ \\
$\mathrm{O} 2-\mathrm{C} 9$ & $1.2255(17)$ & $\mathrm{C} 10-\mathrm{C} 15$ & $1.390(2)$ \\
$\mathrm{O} 3-\mathrm{C} 15$ & $1.3626(19)$ & $\mathrm{C} 10-\mathrm{C} 11$ & $1.392(2)$ \\
$\mathrm{O} 3-\mathrm{C} 16$ & $1.4151(19)$ & $\mathrm{C} 11-\mathrm{C} 12$ & $1.383(2)$ \\
$\mathrm{O} 4-\mathrm{C} 13$ & $1.3636(18)$ & $\mathrm{C} 12-\mathrm{C} 13$ & $1.391(2)$ \\
$\mathrm{O} 4-\mathrm{C} 17$ & $1.432(2)$ & $\mathrm{C} 12-\mathrm{H} 12 \mathrm{~A}$ & 0.9300 \\
$\mathrm{O} 5-\mathrm{C} 11$ & $1.3651(18)$ & $\mathrm{C} 13-\mathrm{C} 14$ & $1.385(2)$ \\
$\mathrm{O} 5-\mathrm{C} 18$ & $1.419(2)$ & $\mathrm{C} 14-\mathrm{C} 15$ & $1.384(2)$ \\
$\mathrm{C} 1-\mathrm{C} 2$ & $1.371(2)$ & $\mathrm{C} 14-\mathrm{H} 14 \mathrm{~A}$ & 0.9300 \\
$\mathrm{C} 1-\mathrm{C} 6$ & $1.403(2)$ & $\mathrm{C} 16-\mathrm{H} 16 \mathrm{~A}$ & 0.9600 \\
$\mathrm{C} 1-\mathrm{H} 1 \mathrm{~A}$ & 0.9300 & $\mathrm{C} 16-\mathrm{H} 16 \mathrm{~B}$ & 0.9600
\end{tabular}




\begin{tabular}{|c|c|c|c|}
\hline $\mathrm{C} 2-\mathrm{C} 3$ & $1.392(3)$ & $\mathrm{C} 16-\mathrm{H} 16 \mathrm{C}$ & 0.9600 \\
\hline $\mathrm{C} 2-\mathrm{H} 2 \mathrm{~A}$ & 0.9300 & C17-H17A & 0.9600 \\
\hline $\mathrm{C} 3-\mathrm{C} 4$ & $1.381(2)$ & C17-H17B & 0.9600 \\
\hline C3- 19 & $1.511(2)$ & $\mathrm{C} 17-\mathrm{H} 17 \mathrm{C}$ & 0.9600 \\
\hline $\mathrm{C} 4-\mathrm{C} 5$ & $1.387(2)$ & C18-H18A & 0.9600 \\
\hline $\mathrm{C} 4-\mathrm{H} 4 \mathrm{~A}$ & 0.9300 & $\mathrm{C} 18-\mathrm{H} 18 \mathrm{~B}$ & 0.9600 \\
\hline $\mathrm{C} 5-\mathrm{C} 6$ & $1.3998(19)$ & $\mathrm{C} 18-\mathrm{H} 18 \mathrm{C}$ & 0.9600 \\
\hline $\mathrm{C} 6-\mathrm{C} 7$ & $1.446(2)$ & C19-H19A & 0.9600 \\
\hline $\mathrm{C} 7-\mathrm{C} 8$ & $1.340(2)$ & C19-H19B & 0.9600 \\
\hline C7-H7A & 0.9300 & $\mathrm{C} 19-\mathrm{H} 19 \mathrm{C}$ & 0.9600 \\
\hline $\mathrm{C} 8-\mathrm{C} 9$ & $1.441(2)$ & & \\
\hline $\mathrm{C} 5-\mathrm{O} 1-\mathrm{H} 1 \mathrm{~B}$ & 109.5 & $\mathrm{C} 11-\mathrm{C} 12-\mathrm{C} 13$ & $118.68(15)$ \\
\hline $\mathrm{C} 15-\mathrm{O} 3-\mathrm{C} 16$ & $119.21(13)$ & $\mathrm{C} 11-\mathrm{C} 12-\mathrm{H} 12 \mathrm{~A}$ & 120.7 \\
\hline $\mathrm{C} 13-\mathrm{O} 4-\mathrm{C} 17$ & $117.54(14)$ & $\mathrm{C} 13-\mathrm{C} 12-\mathrm{H} 12 \mathrm{~A}$ & 120.7 \\
\hline $\mathrm{C} 11-\mathrm{O} 5-\mathrm{C} 18$ & $118.63(14)$ & $\mathrm{O} 4-\mathrm{C} 13-\mathrm{C} 14$ & $123.74(14)$ \\
\hline $\mathrm{C} 2-\mathrm{C} 1-\mathrm{C} 6$ & $121.65(15)$ & $\mathrm{O} 4-\mathrm{C} 13-\mathrm{C} 12$ & $114.86(14)$ \\
\hline $\mathrm{C} 2-\mathrm{C} 1-\mathrm{H} 1 \mathrm{~A}$ & 119.2 & $\mathrm{C} 14-\mathrm{C} 13-\mathrm{C} 12$ & $121.39(14)$ \\
\hline $\mathrm{C} 6-\mathrm{C} 1-\mathrm{H} 1 \mathrm{~A}$ & 119.2 & $\mathrm{C} 15-\mathrm{C} 14-\mathrm{C} 13$ & $118.62(14)$ \\
\hline $\mathrm{C} 1-\mathrm{C} 2-\mathrm{C} 3$ & $120.75(15)$ & $\mathrm{C} 15-\mathrm{C} 14-\mathrm{H} 14 \mathrm{~A}$ & 120.7 \\
\hline $\mathrm{C} 1-\mathrm{C} 2-\mathrm{H} 2 \mathrm{~A}$ & 119.6 & $\mathrm{C} 13-\mathrm{C} 14-\mathrm{H} 14 \mathrm{~A}$ & 120.7 \\
\hline $\mathrm{C} 3-\mathrm{C} 2-\mathrm{H} 2 \mathrm{~A}$ & 119.6 & $\mathrm{O} 3-\mathrm{C} 15-\mathrm{C} 14$ & $123.97(13)$ \\
\hline $\mathrm{C} 4-\mathrm{C} 3-\mathrm{C} 2$ & $118.51(15)$ & $\mathrm{O} 3-\mathrm{C} 15-\mathrm{C} 10$ & $114.45(13)$ \\
\hline $\mathrm{C} 4-\mathrm{C} 3-\mathrm{C} 19$ & $120.07(17)$ & $\mathrm{C} 14-\mathrm{C} 15-\mathrm{C} 10$ & $121.57(14)$ \\
\hline $\mathrm{C} 2-\mathrm{C} 3-\mathrm{C} 19$ & $121.41(16)$ & $\mathrm{O} 3-\mathrm{C} 16-\mathrm{H} 16 \mathrm{~A}$ & 109.5 \\
\hline $\mathrm{C} 3-\mathrm{C} 4-\mathrm{C} 5$ & $121.08(15)$ & $\mathrm{O} 3-\mathrm{C} 16-\mathrm{H} 16 \mathrm{~B}$ & 109.5 \\
\hline $\mathrm{C} 3-\mathrm{C} 4-\mathrm{H} 4 \mathrm{~A}$ & 119.5 & $\mathrm{H} 16 \mathrm{~A}-\mathrm{C} 16-\mathrm{H} 16 \mathrm{~B}$ & 109.5 \\
\hline $\mathrm{C} 5-\mathrm{C} 4-\mathrm{H} 4 \mathrm{~A}$ & 119.5 & $\mathrm{O} 3-\mathrm{C} 16-\mathrm{H} 16 \mathrm{C}$ & 109.5 \\
\hline $\mathrm{O} 1-\mathrm{C} 5-\mathrm{C} 4$ & $121.92(13)$ & $\mathrm{H} 16 \mathrm{~A}-\mathrm{C} 16-\mathrm{H} 16 \mathrm{C}$ & 109.5 \\
\hline $\mathrm{O} 1-\mathrm{C} 5-\mathrm{C} 6$ & $117.19(12)$ & $\mathrm{H} 16 \mathrm{~B}-\mathrm{C} 16-\mathrm{H} 16 \mathrm{C}$ & 109.5 \\
\hline $\mathrm{C} 4-\mathrm{C} 5-\mathrm{C} 6$ & $120.89(13)$ & $\mathrm{O} 4-\mathrm{C} 17-\mathrm{H} 17 \mathrm{~A}$ & 109.5 \\
\hline $\mathrm{C} 5-\mathrm{C} 6-\mathrm{C} 1$ & $117.12(14)$ & $\mathrm{O} 4-\mathrm{C} 17-\mathrm{H} 17 \mathrm{~B}$ & 109.5 \\
\hline $\mathrm{C} 5-\mathrm{C} 6-\mathrm{C} 7$ & $118.98(12)$ & $\mathrm{H} 17 \mathrm{~A}-\mathrm{C} 17-\mathrm{H} 17 \mathrm{~B}$ & 109.5 \\
\hline $\mathrm{C} 1-\mathrm{C} 6-\mathrm{C} 7$ & $123.89(13)$ & $\mathrm{O} 4-\mathrm{C} 17-\mathrm{H} 17 \mathrm{C}$ & 109.5 \\
\hline $\mathrm{C} 8-\mathrm{C} 7-\mathrm{C} 6$ & $128.71(13)$ & $\mathrm{H} 17 \mathrm{~A}-\mathrm{C} 17-\mathrm{H} 17 \mathrm{C}$ & 109.5 \\
\hline $\mathrm{C} 8-\mathrm{C} 7-\mathrm{H} 7 \mathrm{~A}$ & 115.6 & $\mathrm{H} 17 \mathrm{~B}-\mathrm{C} 17-\mathrm{H} 17 \mathrm{C}$ & 109.5 \\
\hline $\mathrm{C} 6-\mathrm{C} 7-\mathrm{H} 7 \mathrm{~A}$ & 115.6 & $\mathrm{O} 5-\mathrm{C} 18-\mathrm{H} 18 \mathrm{~A}$ & 109.5 \\
\hline $\mathrm{C} 7-\mathrm{C} 8-\mathrm{C} 9$ & $122.77(13)$ & $\mathrm{O} 5-\mathrm{C} 18-\mathrm{H} 18 \mathrm{~B}$ & 109.5 \\
\hline $\mathrm{C} 7-\mathrm{C} 8-\mathrm{H} 8 \mathrm{~A}$ & 118.6 & $\mathrm{H} 18 \mathrm{~A}-\mathrm{C} 18-\mathrm{H} 18 \mathrm{~B}$ & 109.5 \\
\hline $\mathrm{C} 9-\mathrm{C} 8-\mathrm{H} 8 \mathrm{~A}$ & 118.6 & $\mathrm{O} 5-\mathrm{C} 18-\mathrm{H} 18 \mathrm{C}$ & 109.5 \\
\hline $\mathrm{O} 2-\mathrm{C} 9-\mathrm{C} 8$ & $122.17(14)$ & $\mathrm{H} 18 \mathrm{~A}-\mathrm{C} 18-\mathrm{H} 18 \mathrm{C}$ & 109.5 \\
\hline $\mathrm{O} 2-\mathrm{C} 9-\mathrm{C} 10$ & $118.36(14)$ & $\mathrm{H} 18 \mathrm{~B}-\mathrm{C} 18-\mathrm{H} 18 \mathrm{C}$ & 109.5 \\
\hline $\mathrm{C} 8-\mathrm{C} 9-\mathrm{C} 10$ & $119.47(12)$ & $\mathrm{C} 3-\mathrm{C} 19-\mathrm{H} 19 \mathrm{~A}$ & 109.5 \\
\hline $\mathrm{C} 15-\mathrm{C} 10-\mathrm{C} 11$ & $118.35(13)$ & $\mathrm{C} 3-\mathrm{C} 19-\mathrm{H} 19 \mathrm{~B}$ & 109.5 \\
\hline $\mathrm{C} 15-\mathrm{C} 10-\mathrm{C} 9$ & $120.27(13)$ & $\mathrm{H} 19 \mathrm{~A}-\mathrm{C} 19-\mathrm{H} 19 \mathrm{~B}$ & 109.5 \\
\hline $\mathrm{C} 11-\mathrm{C} 10-\mathrm{C} 9$ & $121.37(13)$ & $\mathrm{C} 3-\mathrm{C} 19-\mathrm{H} 19 \mathrm{C}$ & 109.5 \\
\hline $\mathrm{O} 5-\mathrm{C} 11-\mathrm{C} 12$ & $124.18(14)$ & $\mathrm{H} 19 \mathrm{~A}-\mathrm{C} 19-\mathrm{H} 19 \mathrm{C}$ & 109.5 \\
\hline $\mathrm{O} 5-\mathrm{C} 11-\mathrm{C} 10$ & $114.43(13)$ & $\mathrm{H} 19 \mathrm{~B}-\mathrm{C} 19-\mathrm{H} 19 \mathrm{C}$ & 109.5 \\
\hline
\end{tabular}




$$
\begin{aligned}
& \mathrm{C} 12-\mathrm{C} 11-\mathrm{C} 10 \\
& \mathrm{C} 6-\mathrm{C} 1-\mathrm{C} 2-\mathrm{C} 3 \\
& \mathrm{C} 1-\mathrm{C} 2-\mathrm{C} 3-\mathrm{C} 4 \\
& \mathrm{C} 1-\mathrm{C} 2-\mathrm{C} 3-\mathrm{C} 19 \\
& \mathrm{C} 2-\mathrm{C} 3-\mathrm{C} 4-\mathrm{C} 5 \\
& \mathrm{C} 19-\mathrm{C} 3-\mathrm{C} 4-\mathrm{C} 5 \\
& \mathrm{C} 3-\mathrm{C} 4-\mathrm{C} 5-\mathrm{O} 1 \\
& \mathrm{C} 3-\mathrm{C} 4-\mathrm{C} 5-\mathrm{C} 6 \\
& \mathrm{O} 1-\mathrm{C} 5-\mathrm{C} 6-\mathrm{C} 1 \\
& \mathrm{C} 4-\mathrm{C} 5-\mathrm{C} 6-\mathrm{C} 1 \\
& \mathrm{O} 1-\mathrm{C} 5-\mathrm{C} 6-\mathrm{C} 7 \\
& \mathrm{C} 4-\mathrm{C} 5-\mathrm{C} 6-\mathrm{C} 7 \\
& \mathrm{C} 2-\mathrm{C} 1-\mathrm{C} 6-\mathrm{C} 5 \\
& \mathrm{C} 2-\mathrm{C} 1-\mathrm{C} 6-\mathrm{C} 7 \\
& \mathrm{C} 5-\mathrm{C} 6-\mathrm{C} 7-\mathrm{C} 8 \\
& \mathrm{C} 1-\mathrm{C} 6-\mathrm{C} 7-\mathrm{C} 8 \\
& \mathrm{C} 6-\mathrm{C} 7-\mathrm{C} 8-\mathrm{C} 9 \\
& \mathrm{C} 7-\mathrm{C} 8-\mathrm{C} 9-\mathrm{O} 2 \\
& \mathrm{C} 7-\mathrm{C} 8-\mathrm{C} 9-\mathrm{C} 10 \\
& \mathrm{O} 2-\mathrm{C} 9-\mathrm{C} 10-\mathrm{C} 15 \\
& \mathrm{C} 8-\mathrm{C} 9-\mathrm{C} 10-\mathrm{C} 15 \\
& \mathrm{O} 2-\mathrm{C} 9-\mathrm{C} 10-\mathrm{C} 11 \\
& \mathrm{C} 8-\mathrm{C} 9-\mathrm{C} 10-\mathrm{C} 11 \\
&
\end{aligned}
$$

$121.39(14)$

$-0.3(3)$
$0.4(2)$
$-179.49(16)$
$-0.5(2)$
$179.37(14)$
$179.78(14)$
$0.5(2)$
$-179.66(13)$
$-0.3(2)$
$1.36(19)$
$-179.32(13)$
$0.2(2)$
$179.15(14)$
$-179.68(14)$
$1.4(2)$
$-179.96(14)$
$179.67(15)$
$-0.8(2)$
$75.90(19)$
$-103.68(16)$
$-103.56(17)$
$76.87(19)$

$5.6(2)$

$-174.96(14)$

$-179.09(12)$

$0.4(2)$

$0.4(2)$

179.85 (13)

$179.48(13)$

$0.1(2)$

$0.2(2)$

$-179.57(15)$

$179.14(13)$

$-0.6(2)$

$-178.99(14)$

$0.8(2)$

$3.0(2)$

$-178.38(15)$

$178.26(14)$

$-0.3(2)$

$-178.96(13)$

$1.6(2)$

$-0.3(2)$

$-179.73(13)$

Hydrogen-bond geometry $\left(\AA,{ }^{\circ}\right)$

\begin{tabular}{lllll}
\hline$D-\mathrm{H} \cdots A$ & $D-\mathrm{H}$ & $\mathrm{H} \cdots A$ & $D \cdots A$ & $D-\mathrm{H} \cdots A$ \\
\hline $\mathrm{O} 1-\mathrm{H} 1 B \cdots \mathrm{O} 2^{\mathrm{i}}$ & 0.82 & 1.88 & $2.6653(15)$ & 161 \\
$\mathrm{C} 17-\mathrm{H} 17 A \cdots \mathrm{O} 1^{\mathrm{ii}}$ & 0.96 & 2.70 & $3.520(2)$ & 144 \\
\hline
\end{tabular}

Symmetry codes: (i) $x, y-1, z$; (ii) $-x+1,-y,-z$. 